\title{
Leather of the tilapia, how healing in the treatment of burns
}

\begin{abstract}
The objective of this article is to analyze and investigate the extent of the use of tilapia skin in the treatment of burns, especially in the region of the hands. Burns are skin lesions due to a trauma of thermal origin, which may be severe or not, which may be beyond the skin, tendons, and bones. This treatment, inspired by the research of Brazilian doctors, This treatment, inspired by the research of Brazilian doctors of the federal university of the state of Ceara, located in the north of Brazil, have already been tested, and all the samples have been analyzed. To finally find the final result of analysis, noting that fish skin has a collagen very close to human skin and suitable for a treatment function but quick and pain-free for the patient who is receiving this treatment emerged in order to provide a better recovery of the patients visando the low costs, a process of healing more agile that diminishes the risk of contamination of the wound as well as to obtain better aesthetic results and the social welfare, since normally the one of the fish is discarded, it seeks to know better this form of treatment and the positive results that it can offer the patient. However, taking Paraguay as a base, it is noted that the treatment model would bring positive results for this research to be used, since a place where many people live with low income, and community, treatment with low cost, and with many benefits for who receives it. With regard to the method of approach of each degree of burn, research will be used to expose the physiology of human skin, thiopathology of different degrees of burns as well as the evaluation of each, in addition to preventive methods.
\end{abstract}

Keywords: burns, treatment, cicatrization, methods, physiology, human skin, community, treatment, income, healing, patients, preventive methods, expose
Volume 6 Issue 4 - 2019

\author{
Alexander Zahr Lordello,' Amanda Guimaraes \\ Camargo Bianco,' Ariela Mosmann Ribas,' \\ Chayenne Gomes Bueno Arantes, Eduardo \\ Severo Monteiro,' Elenice Pedroso da Silva, ${ }^{2}$ \\ Elissandra Nascimento da Costa, ${ }^{3}$ Eloisa \\ Christini Beutler, Fabio setti Ximenes,' \\ Fernanda Waitman de Oliveira Silva,' Francisco \\ Deleon Oliveira dos Santos, Lays Alexandre \\ Bezerra, Maicon Baudouin Mazzo, ${ }^{4}$ Matheus \\ Zahr Geronimo' \\ 'Medical Student, Polytechnic and Artistic University of Paraguay, \\ Paraguay \\ ${ }^{2}$ Nurse at Unip University and Medical Student by Polytechnic \\ University and Artistic Paraguay, Paraguay \\ ${ }^{3}$ Nurse from the FINCA University, Medical Student by the \\ Polytechnic and Artistic University of Paraguay, Paraguay \\ ${ }^{4}$ Physiotherapist, Institute of Higher Education of Alagoas and \\ Medical Student, Polytechnic and Artistic University of Paraguay, \\ Paraguay
}

Correspondence: Amanda Guimaraes Camargo Bianco, Medica Student, Polytechnic and Artistic University of Paraguay, Paraguay, Email Amandacamargob@outlook.com

Received: August 10,2019 | Published: August 30, 2019

\section{Introduction}

Burns are cutaneous lesions or other tissues, usually caused by trauma thermal origin which may be serious or not. Burns are classified according to the depth: 1st, 2nd and 3rd grade, third grade being the most serious. Due to the high degree of complexity, time, pain and costs of conventional treatment, coroners Brazilians looked for a way to treat injuries so that faster healing occurs, decrease the risk of wound contamination, accessible to needy people for better cosmetic results.

Thus, doctors went to experience skin tilapia - traditional Brazilian fish - as a method of treatment of burns of second and third degree. This unconventional treatment is bringing positive results in patients.

The focus of the work will be in the region of hand, with all the injuries in all degrees of burns. And regarding the method of approach, research will be used to expose the physiology of human skin, hilosiopatología of different degrees of burns and evaluation of each, in addition to the preventive methods.

\section{Purpose}

Publicize and disseminate the evolution of patients with burns region hands with the use of tilapia skin instead of conventional treatments.

\section{Specific objectives}

a) Benefits provided to the patient.

b) The operation of the treatment.

c) Comparison between use of fish skin and conventional dressing.

d) The types of burns and injuries to hands.

\section{Indices burn cases}

\section{Materials and methods}

In this chapter the material used and the procedures performed in the development of all research is presented. Referring next material and procedures described in the following is done.

In its references - as illustrative material, images that have references in the body of work were used. These were inserted at pp. 21.

As a basis for the specific purpose of this research, articles of several countries were used as Norway, USA, Brazil, among others. And justifying the use of such bibliographic activities, by having those states, and effectively applied the most palpable in this article material tilapia skin as a means of treating burns. 
As the model makes references and incorporates the entire development of this thesis was used: EVA (ethylene vinyl acetate), Plasticine (composed of calcium carbonate, petrolatum wax and acid to stabilize the mixture).

In the main structure of the model - which are mainly hands it was created papier-mâché (more recycled paper tail).

Today, the possibilities of paper mache have been rediscovered by artists, interior decorators and fashion designers. The material has found its way into modern and sophisticated decoration; it is used for jewelry and fashion accessories, even for suits.

In the same structure, gouache ink (binder mixture or gum arabic with white pigment) was used.

In sum, the methods and materials served for this academic sum are cited in this aspect in the work body. Later the interested reader will get a better understanding of the purpose and main objective of this activity.

\section{Theoretical foundations}

Burns are skin lesions or other tissues, usually caused by heat, electricity. Burns can be classified according to the depth: $1^{\circ}, 2^{\circ}$ and $3^{\circ}$. A burn, treated with the conventional method, it takes up to eight weeks to heal, leaving permanent marks.

Thus, medical researchers from Ceará in Brazil, went to experience skin tilapia - fish traditional Brazilian method of treatment of burns of second and third degree. Exotic and unconventional treatment is bringing positive results.

\section{Burns}

Burns are guilty of more serious trauma that can happen to a human being. Plus fewer acres million people are disabled each year worldwide due to burns. They can be differentiated by their type (thermal burns, chemical, and electricity) and classification (1st grade, 2nd grade, 3 rd grade). These factors act on the lining of the human body, determining the partial or total destruction of the skin and its attachments, can reach deeper layers, as subcutaneous fat, muscles, tendons and bones.

\section{First grade burns}

Call scorch are those involving only the epidermis, the outermost layer of the skin. Symptoms include severe pain and local redness, but with pale skin when touched. Generally improve in the range of 3 to 6 days and can exfoliate and leave no sequelae (Figure $1 \& 2$ ).

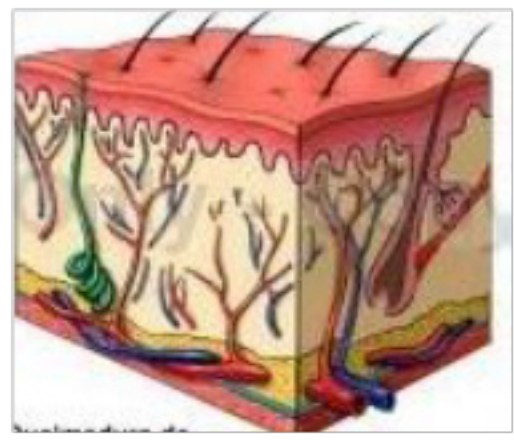

Figure I First degree burn, reaching only the superficial layers of the epidermis.

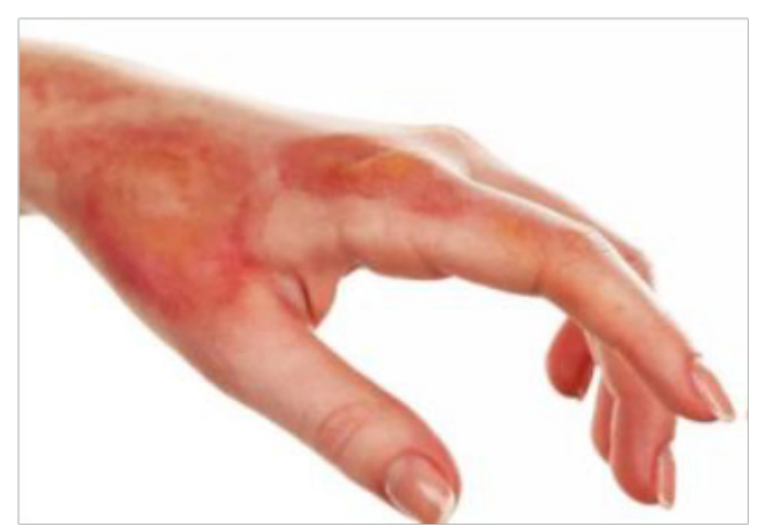

Figure 2 Part of the hand that was reached by a first degree burn.

\section{Second degree burns}

It is currently divided into 2nd surface degree and 2nd degree deep. Burn 2nd degree surface it is one that involves the epidermis and the superficial portion of the dermis. The symptoms are the same as the first degree burn, including the appearance of bubbles and a wet appearance of the lesion. Healing is longer than can take up to 3 weeks, usually no scarring but the site of injury may be clearer.

Burns deep second degree is those rushing entire dermis, being similar to 3rd degree burns. As there is a risk of destruction of nerve endings in the skin, this type of burn, which is much more serious, it may even be less painful than superficial burns. Sweat glands and hair follicles can also be destroyed, causing the skin to become dry and lose their hair. Healing takes longer than 3 weeks and often leave scars (Figure $3 \& 4$ ).

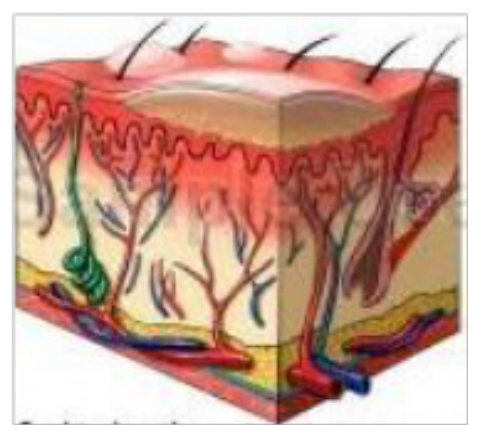

Figure 3 Second degree burn, where the entire epidermis is wrapped and a little of the dermis, can wrap the entire dermis, skin bubbles.

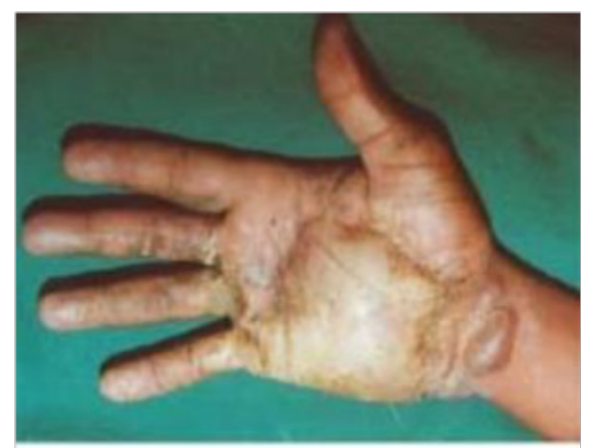

Figure 4 Part of the hand that was reached by a second degree burn. 


\section{Third degree burns}

Deep burns that affect the dermis and reach all subcutaneous tissues, with destruction of nerves, hair follicles, sweat glands and capillaries, may even reach muscle and bone structures. They are whitish/greyish, dry, painless and disfiguring lesions that do not heal without surgical support, needing grafts (Figure $5 \& 6$ ).

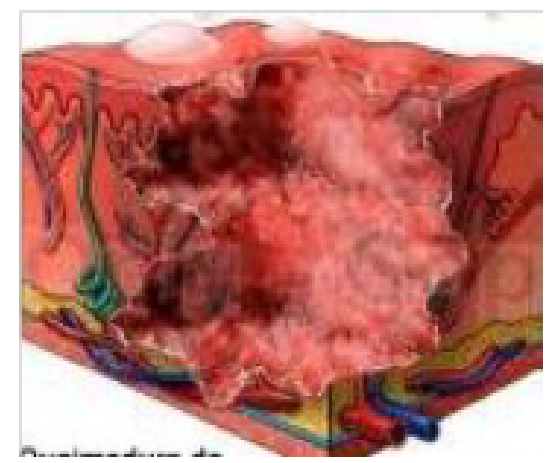

Figure 5 Part of the hand that was reached by a second degree burn.

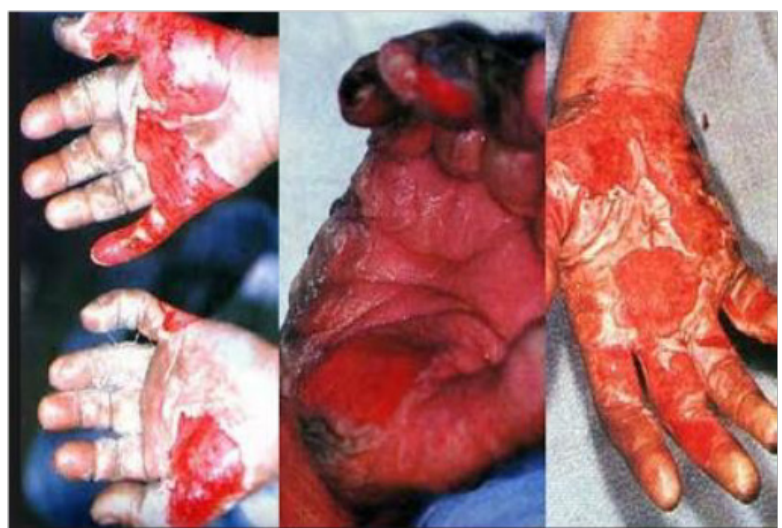

Figure 6 Part of the hand that was reached by a second third degree burn.

\section{Extension burn}

In addition to the depth of the burn, it is also important the extent of injury. All patients with lesions 2nd or 3rd grade must be assessed in relation to the percentage of body area reached (Figure 7).

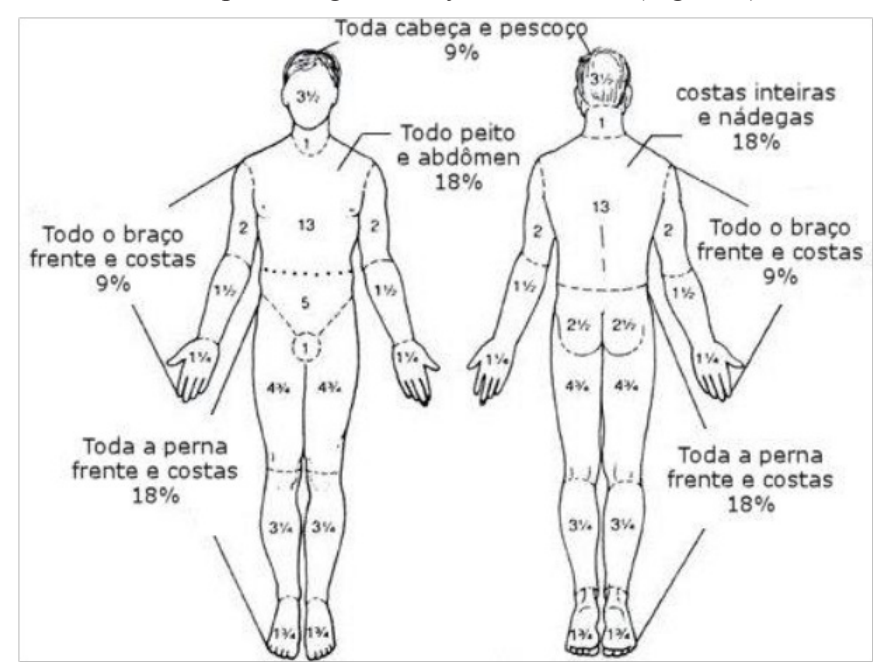

Figure 7 percentage of body area affected.
A severity rating is divided as follows: mild burn, burn moderate and severe sun burn.

\section{Slight burn}

Less than $10 \%$ of the body surface of an adult with 2 nd degree burns.

Less than $5 \%$ of the body surface of a child or elderly with 2 nd degree burns.

Less than $2 \%$ of the body surface burn 3rd degree.

\section{Moderate burn}

1. 10 to $20 \%$ of the body surface of an adult with 2 nd degree burns.

2. 5 to $10 \%$ of the body surface of a child or elderly with 2 nd degree burns.

3. 2 to $5 \%$ of the body surface burn 3 rd degree.

\section{Burn grave}

a. Over $20 \%$ of the body surface of an adult with 2 nd degree burns

b. More than $10 \%$ of the body surface of a child or elderly 2 nd degree burns

c. More than $5 \%$ of the body surface burn 3 rd grade

d. High-voltage electrical burns

e. Burns proven respiratory tract by inhalation of hot air

f. Significant burns to the face, eyes, ears, genitals or joints

g. Other serious injuries associated with sunburn, and traumas such as fractures.

\section{First care}

Each type of burn requires a special and specific care, depending on the causative agent, the extent and depth of the injury, but overall, the first aid care, and if necessary the victim is directed to a patient post or hospital care.

The first and primary care for all the next steps to do exactly happen then, when a normal wound contains half 60,000 to 80,000 bacteria per gram of tissue, and in the first 48 hours proliferate at a level of 10 million organisms per gram of tissue. Pruit and Mc Manus checked data that infections from burns fu the leading cause of death. ${ }^{1}$

Merrell and Asoc report that the decline in mortality from bacterial infection only to have the correct treatment occurs. ${ }^{2}$

\section{Etiology, epidemiology and preventive measures}

The most common causes of burns are a flame of fire, contact with boiling water or other hot liquids and contact with heated objects. Less common are burns caused by the electric current, transformed into heat contact with the body. Chemical burn is misnomer given to caustic injury caused by chemical agents, wherein the tissue damage is not always heat production. Burns in children, in most cases occur in the home environment and are caused by the spilling of hot liquid on the body, like boiling water in the kitchen, hot water bath, drinks and other hot liquids like kitchen oil. 
In these cases they tend to be more superficial, but more extensive. It is still common cause of burns, in Brazil, the flame of fire by liquid handling ethyl alcohol, responsible for most cases in adolescents and second leading cause in children treated at the referral hospital in urgency of Minas Gerais and 40 burns\% of children between seven and 11 years old at a teaching hospital in the state of São Paulo. In contrast, adults are more often burned with flaming fire, and above all, professional environment. The resulting burns, therefore, tend to be deeper and usually accompanied by damage caused by smoke inhalation.

Burns through contact with hot surfaces such as ovens, toasters, grills and heaters, items are more common in individual's seizures, alcohol or under the influence of illicit drugs and elderly during episodes of loss of consciousness disease. They also tend to be deeper due to prolonged contact with the heat source Electrical injuries can be caused by the passage of electric current through the body or by exposure to heat generated by the arc current high tension. In the first case, besides thermal injury, there is a risk of impaired, which must be properly monitored cardiac electrical conduction.

Each year in the UK 250,000 people suffer burns, 175 thousand are treated in emergency units, 13 thousand are hospitalized, and 1000 electrolyte resuscitation measures are needed and 300 results in death. However, the numbers are even more alarming in developing countries, such as Nepal, where mortality can reach 17 times the British rate. According to the Brazilian Society of Burns, in Brazil a million burn cases occur each year, 200,000 are treated in emergency services, and 40 thousand require hospitalization.

Burns are among the leading external causes of death recorded in Brazil, losing only to other violent causes, including transportation accidents and homicides. Federal District conducted in the study showed mortality rate of $6.2 \%$ among those burned in the hospital emergency. In response to recent attacks and terrorist threats, especially in the United States, it was strategic and logistic systems, encompassing well-trained teams to pay attention mass in catastrophic events developed, including victims burns.

Based on the assumption that $90 \%$ of burns could be prevented, preventive measures are imposed to reduce its incidence and depend on education and legislation. Educational prevention measures consist in guiding early children to avoid a risk of burns in the domestic environment, in including in school curricula the teaching prevention of accidents, including burns, as well as general preventive campaigns the entire population.

Private educational campaigns, to be effective, must be based on reliable epidemiological data that identify specific causes of burns and populations at risk, which must be periodically addressed. The legislation basically contains the mandatory measures installation of fire prevention equipment in public and private buildings as well as specific safety equipment at work.

In our environment, a legal measure of extreme importance was the prohibition on marketing the liquid ethyl alcohol, by resolution of the National Health Surveillance Agency, but has still met with resistance from producers, who through the limits.

Justice obtained insists on maintaining product distribution in commerce in general. Unfortunately all prevention strategies implemented have not yet been able to produce the expected impact in the dramatic epidemiological picture of burns.

\section{Physiopathology of burns}

Burn compromises the functional integrity of the skin, responsible for the electrolyte homeostasis, the internal temperature control, flexibility and lubrication of the body surface. The magnitude of the commitment of these functions depends on the extent and depth of the burn.

Thermal injury in the body causes a local response, translated by coagulation necrosis of tissue and progressive thrombosis adjacent vessels over a period of 12 to 48 hours. The burn wound at first is sterile, but necrotic tissue rapidly becomes colonized by endogenous and exogenous bacteria producing protease, leading to liquefaction and separation of eschar, resulting in granulation tissue responsible for healing wound, characterized by high shrink ability and fibrosis in third degree burns grau.

In large burns, besides the local response, thermal damage triggers a systemic response of the organism as a result of the release of mediators from the damaged tissue. Damage occurs extensive capillary integrity, with accelerated fluid loss, either by evaporation through the wound or sequestered in the interstices, which is aggravated by byproducts bacterial colonization. In addition, extensive burns, over $40 \%$ of body area, the immune system is unable to define infection, which, systematizing makes rare to come upon cases.

This systemic response is manifested by fever, hyperdynamic circulation and metabolism accelerated pace with increased muscle catabolism resulting from alteration of hypothalamic function of (increasing glucagon secretion, cortisol, and catecholamines), deficiency of the gastrointestinal barrier ( passage of bacteria and their by-products to the systemic circulation), bacterial contamination of the burned area (systemic release of bacteria and by-products), the heat loss (evaporation through the wound which leads to hypothermia) and loss fluids (electrolyte imbalance).

There are many factors involved in the burns to be observed in their assessment. The depth, extent and location of the burn, the age of the victim, the existence of previous diseases, concomitance of aggravating conditions and smoke inhalation have to be considered in assessing burned. The atmosphere of the evaluation must be kept heated; the skin must be discovered and tested in parts, to minimize fluid loss by evaporation.

\section{Depth}

Depends on the intensity of the thermal agent, if generator or heat exchanger, and time of contact with the tissue. It is the determining factor of aesthetic and functional result of the burn and can be evaluated in degrees.

\section{Extension}

General risks of burning in the early hours are crucially dependent on extension of the burned area, with higher systemic effect due to the loss of function of the skin, the higher the affected area. The extension is calculated as a percentage of total body surface area (SC), being considered only burned areas with deep second and third degree.

A practical method for calculating the burned area as a measure reference the palm of the hand victim, whereas the palmar surface, including the fingers together and extended, corresponding to approximately $1 \%$ of their body surface. Excluding the fingers, palmar surface represents $0.5 \% \mathrm{BSA}$, regardless of edad.15,16.grosero, this 
method is very useful in determining if the area immediately, mainly irregular burns exceeds $15 \mathrm{SC} \%$ of adult and $10 \%$ of SC child, a situation in which emergency rehydration should be established. However, for a more accurate assessment of the extent of the burn, the most used method is the rule of nines Wallace easy memorization.

The method should be adjusted for children under 10 years old. more acurado method to assess the burn area used diagram Lund \& Browder, 17.18 which weights the body shape changes according to age. It is, therefore, more suitable for children, but should be available, printed on the card of the victim, given the difficulty of being memorized.

\section{Degree}

a. Only compromises the epidermis.

b. It presents redness, heat and pain.

c. No bubbles.

d. It develops peeling in a few days.

e. It is regulated without scarring.

f. It is regulated without scarring.

g. It is not considered in the assessment of the affected area.

\section{2nd grade}

a. Fully engages the epidermis and dermis partially.

b. You have pain, erythema, edema, erosion or ulceration.

c. There spontaneous regeneration.

d. Epithelialization occurs from the skin appendages (hair follicles and glands).

e. Slower healing (2-4 weeks).

f. It cans sequelae: dyschromia (surface).

g. Scar (deep).

\section{3rd grade}

a. Destroy all skin layers reaching the subcutaneous, which can reach the tendons, ligaments, muscles and bones.

b. Cause white or brown, dry, hard, inelastic injury.

c. It is painless.

d. No spontaneous regeneration, requiring graft.

e. Where appropriate, it can heal, but retraction of the edges.

\section{Anatomy and physiology of skin}

The skin is the largest organ, it covers and protects the body against foreign bodies, still has nerve function constituting the sense of touch, it works by regulating water loss, the homeotasa due to the action of the sweat glands and capillaries blood. When this protection is wholly or partially destroyed due to burns, altered human homeostasis occurs, compromising the individual's survival.

Skin and all the anatomical body compartments have divisions, the epidermis and hypodermis and dermis Annexes as hair follicles.
Epidermis: It is a layer with a different depth as the body region. Areas with increased friction as palms and feet have a thicker layer, and reach up to $2 \mathrm{~mm}$ thick, constituted by a keratinized stratified epithelium paved. The main cell is the keratinocyte, which produces keratin (responsible for protection). Intercalated between the keratinocytes, there are other cell types, such as melanocytes, Langerhan cells and Merkel cells.

Dermis: It is a connective tissue supporting the epidermis. It consists of fibrin collagen and elastin numerous fibrocytes manufacturing these proteins and support tissue, they consist of two main layers papillary by the epidermis and the denser reticular layer is the dermis including the sensor to the heat called Ruffini and free nerve endings sensitive to pain and temperature in the case of a skin burn.

Hypodermis: Is known as the body against cold by being formed by adipose tissue it makes connection with both the dermis and the muscle fascia (reserving energy and temperature model body supeficie, fixing bodies and shock absorption.

Anatomically patients with burns face, hands, feet, perineum and respiratory injuries have increased morbidity, more limited indication of chronic sequelae and mortality phase, special attention to the symptoms of burn is recommended.

\section{Treatment of burns}

Everywhere in the world the burn and known as the most painful way and with a treatment that leaves scars, the Brazilian Society of Burns relates that one of the major causes of death worldwide are burns. $^{3}$

In Brazil the method used to treat burns and using a cream, which lasts 24 hours every day and need to change the bandage, removing the cream wash the burned area, replace and make a new .This bandage treatment done, is effective, but the patient performs many days, and also happens to leave scars on the affected premises.

But after medical researchers from Ceará in Brazil, they went to experience skin tilapia as a method of treatment of burns of second and third degree obtained great positive results.

This treatment is not yet known everywhere, but already used in Brazil as a cheap way without any pain and an effective result.

\section{Burns hands}

At burning hands perhaps less noticed but the most affected of all, hands represent only $6 \%$ of the body surface, but harms and reached a compromising way.

According Kamolz burns involving the hands have a very large percentage, and is more common in men and boys. ${ }^{4}$

The Initial treatment is of great importance for a successful recovery possible. There are three factors to make it happen: the depth of the injury, in the acute care and reconstructive procedures. Three other pathophysiological factors accompanying the thermal burn and need to be carefully monitored: Edema, inflammation and limited movement.

The presence of these factors leads to an alteration in the position of the hand, called clawhand, characterized by the thumb adduction, metacarpophalangeal hyperextension and bending the cuff. If this condition is not treated early, hardening joint and tendon adhesions formation in the member may occur. 
The conventional treatment used is daily healing with occlusive antibacterial healing agents in functional position, lifting member and daily exercises, which serve primarily to treat edema caused by adhesions. This treatment may take days for there to be a significant improvement.

The proposed treatment is believed that the greatest benefit of using tilapia skin burns treatments is due to social benefit because this skin is usually discarded. With tilapia skin you can accelerate the healing process, reduce the need for medications in addition to skin, leaving an aesthetically nicer appearance (Figure 8).

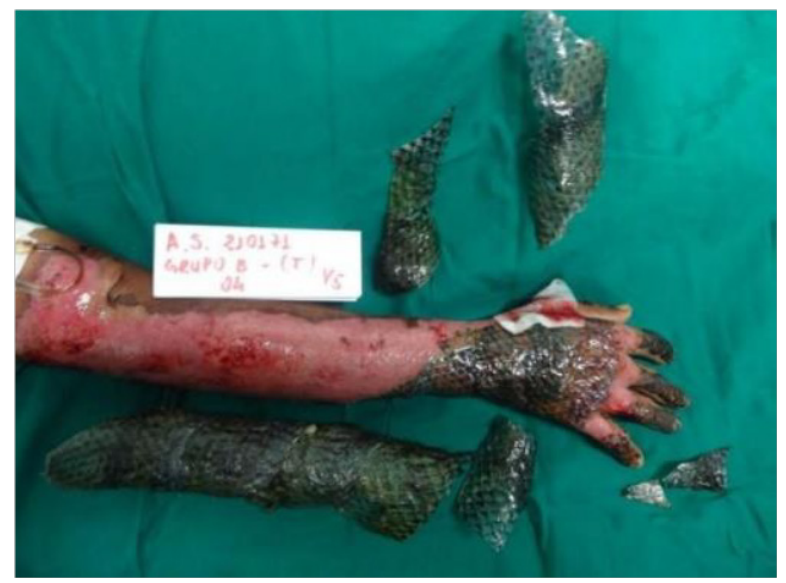

Figure 8 Fish skin treatment to treat burning.

One of the advantages of its use is its high potential adherent to the skin, because there is no need to change it, once placed, fish skin will only be removed at the end of treatment, which lasts on average nine to eleven days. That being the case, the patient does not suffer so much with the change of healing that cause pain and uncomfortable every time it is changed. The research coordinator, doctor Edmar Maciel, explains further efficiency, tilapia skin reduces costs of care. ${ }^{5}$

\section{Benefits of implementation}

Skin tilapia has advantages over pig skin, the advantage of using the skin of tilapia and having a lower possibility of transmitting diseases and has more strength and have a degree of moisture symbolizes human skin so as to facilitate healing in addition to having a high level of collagen is also important in the healing of burns.

Pigskin is $78 \%$ compatibility with human skin and has been used in the treatment of burns. It also contains a large amount of collagen and a similar structure to human skin and can be important in healing human skin in case of burns. One of the major problems of use is the transmission of disease and high cost relative to the skin of tilapia. Odorico de Morais, professor Federal University of Ceará (UFC) tells a little about the product as a social benefit.

\section{Application skin}

First and made clean fish skin with various sterilizing agents, by irradiation after passing kills any virus before the skin is packed and chilled. Once a clean and treated, the skin can last up to two years. And treatment removes any fishy odor (Figure $9 \& 10$ ).

The treatment consists of covering the burned part of the patient's skin with fish skin, and covers it with a bandage, without the need for any cream. After about 10 days, doctors removed the bandage. Tilapia skin, which at that point already dried and loosened burn, can be shelled with the hand.

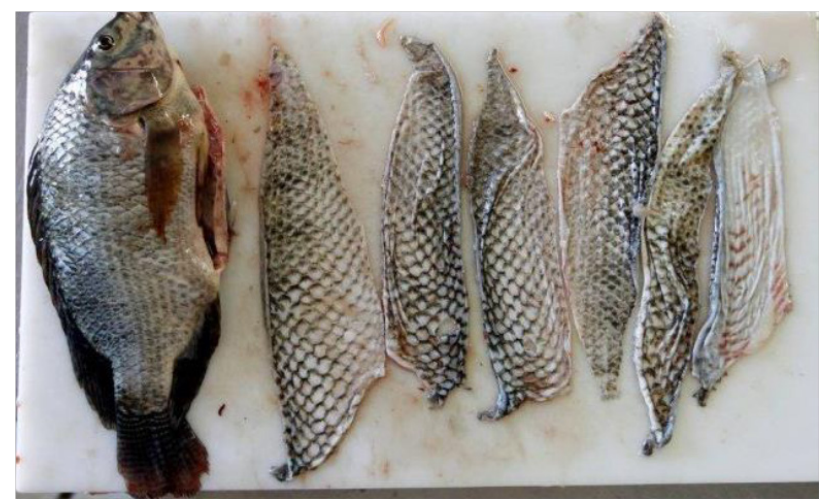

Figure 9 Fish skin removal.

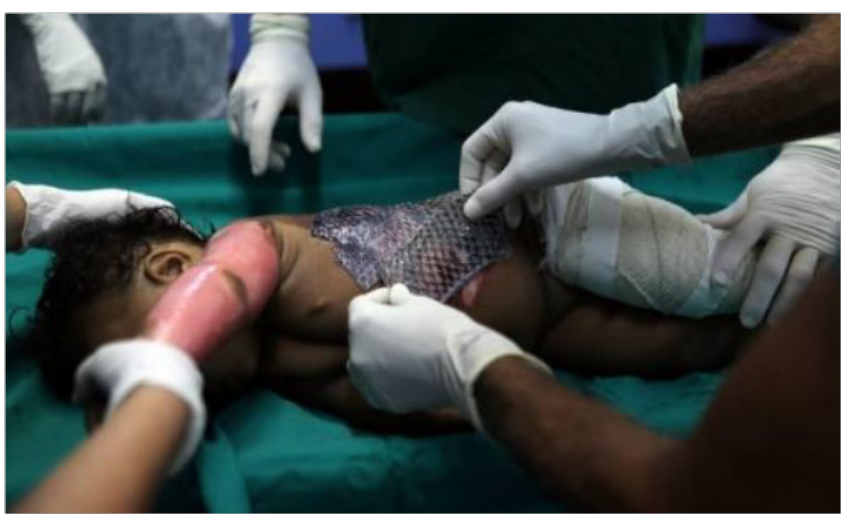

Figure 10 Application of tilapia skin.

\section{Frame indexes in Brazil}

A million people suffer burns each year in the country. Children and low-income people are the biggest victims. The difficulty in treatment and rehabilitation of the burn is the biggest challenge today. Some data Brazilian health ministry said the number of people who are injured with burns.

The figures show that most of these accidents, about $79 \%$ occurs in the home environment and children, from one to nine, are the main victims. Burn treatment with fish skin is still very effective, has rapid healing. This treatment has already been applied in 56 patients with burns of second and third grade in Brazil. As tilapia fish is very common in rivers and nurseries in Brazil, this treatment is fairly inexpensive, allowing a reduction of $75 \%$ of the costs.

Research with this kind of healing unpublished, is being applied to people since 2015. In Brazil there are 46 clinics specializing in burns.

\section{Final thoughts}

Thus, we can conclude that fish skin treatment is a new technique that offers agility in the recovery process and low cost for individuals who have suffered severe burns.

There are many benefits of the use of fish skin in treatments, such as accelerating healing, reducing the use of medications and thus lowering the cost. As she closely resembles human skin, it easily adheres to the area that was affected and there is no need to be changed, only at the end of the treatment, causing less pain and discomfort to the patient.

As it was a technique that was recently discovered, few people used the treatment, more all with rapid recovery and less suffering, since 
the initial treatment is of great importance for a possible successful recovery.

The big question of making a story about all the benefits of tilapia skin, and for everyone to know that such a treatment has such a cheap cost and can be used anywhere, an easy application. Know the importance of this low-cost treatment and, important for low-income people to access this type of treatment.

In the event that scarring that can decrease each time but the chances of sticking with a scar occurs.

Surveys were conducted where a burn hospital was found in Asunción, but a private hospital, which has very high costs in relation to surgeries. If this practice and implemented in public hospitals or clinics that cover low values only for the maintenance of the skins, we reduce pain, rapid healing and many other benefits.

\section{Funding details}

None.

\section{Acknowledgments}

None.

\section{Conflicts of interest}

Authors declare that there is no conflict of interest.

\section{References}

1. Jefferson Braga Silva, Márcio Pereira Lima Ferdinando, John garbin, et al. Initial treatment of hand burns: literature review. Sci Med. 2015;25(2):1-8.

2. Burns. Virtual Health Library. 2015.

3. Everton Carlos Siviero do Vale. Initial management of burns: approach by dermatologists. An Bras Dermatol. 80(1):9-19.

4. Defesa Civil. 2013.

5. Paulo Whitaker e Pablo Garcia. Doctors use fish skin to treat burn victims. 2017. 